\title{
1-P-087 Poster Sessions
}

\section{Antibody drug for control of gingival epithelial cell adhesion}

\section{Mariko Kawai $^{1}$}

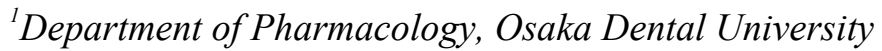

Dental implant therapy is a highly effective treatment for recovering occlusion after tooth loss. An important factor in the success of dental implants is establishing strong osseointegration. If more epithelial cells migrate to the implant bone interface than mesenchymal stem cells, effective osseointegration may fail. Therefore, controlling epithelial cell adhesion and motility would be an effective strategy to increase the success rate of osseointegration. Laminin-332 is a major component of the basement membrane and is composed of three chains ( $\alpha 3, \beta 3$ and $\gamma 2$ ). It is well-known that laminin-332 regulates cellular functions such as adhesion, proliferation, apoptosis and differentiation. These biological functions depend on changes in the structural arrangement of laminin-332 by proteolytic cleavage. We focused on cleavage site of $\alpha 3$ and developed antibodies that target the cleavage site. To investigate the influence of the monoclonal antibody on the cell adhesion function of epithelial cells for the $\alpha 3$ chain of laminin-332, we compared it with the cell adhesion function of human epithelial cells from the Cas9-22 cell line. The monoclonal antibody significantly decreased cell adhesion for the laminin-332 $\alpha 3$ chain when compared with no monoclonal antibody in both laminin-332 doses, 1 and $10 \mu \mathrm{g} / \mathrm{mL}$. We could propose that it would be possible that we change the biological function of laminin-332 to control cell adhesion for the purpose of regulating dental implant therapy. 